... Elisabeth Kurth: erste Hebamme mit einem Schweizer Doktortitel, Lehrbeauftragte an der ZHAW und Geschäftsführerin eines Hebammen-Netzwerkes in Basel

\title{
«Ein Meilenstein für den ganzen Berufsstand»
}

\section{Daniel Lüthi}

Text und Bilder

danielluethi[at]gmx.ch
Vor dem Haus steht ihr Geschäftsfahrzeug, liebevoll nennt sie es «mein Hebammenvelo». Damit fährt sie auf Hausbesuche bei Familien mit Neugeborenen. Ihr Büro befindet sich im Untergeschoss eines eher tristen Wohnblocks. Es gehört zum Schweizerischen Tropen- und Public Health-Institut und sie teilt es mit Kolleginnen aus der Forschung. Keine Spur also von akademischem Schick. Und trotzdem ist diese Frau die erste Hebamme, die sich an einer Schweizer Universität einen Doktortitel erworben hat. «Ja, ich bin schon eine Pionierin», kommentiert Elisabeth Kurth.

\section{Früher Entscheid}

Dass sie Hebamme werden will, wusste sie schon als 17-Jährige. Ein Austauschjahr brachte sie nach Dänemark, zu einer Gastmutter, die in einem Spital arbeitete. Und weil die Berufswahl gerade ein Thema war, durfte sie dort schnuppen - und ihre erste Geburt erleben. «Ich war sehr berührt», erzählt sie, «schwebte wie auf einer Wolke. Und ich spürte, dass ich Frauen in einem solchen Prozess unterstützen will.» Thema ihrer Matura-Arbeit war dann «Mutter werden».
Elisabeth Kurth machte ihre ersten Interviews mit Betroffenen. «Damals begann ich wahrscheinlich mit meiner Forschungsarbeit.» Ihre Gymnasiallehrer seien enttäuscht gewesen, hätten sie lieber in einem Medizinstudium gesehen. Der Griechisch-Lehrer habe sich immerhin durch den Hinweis trösten lassen, dass die Mutter von Sokrates auch Hebamme war. Klar war für Elisabeth Kurth schon damals zweierlei: dass Hebamme keineswegs ein untergeordneter Assistenz-Beruf ist, und dass sie auf ihrem beruflichen Weg auch intellektuelle Herausforderungen suchen würde. «Ich spürte, dass das Hebamme-Sein eine hochkomplexe Tätigkeit mit grossem Potential ist, die auch einen akademischen Hintergrund braucht und verdient hat.»

Vorerst war sie wieder offiziell «Schülerin», nicht Studierende. «In der Hebammenschule war die praktische Ausbildung gut», kommentiert sie im Rückblick, «aber Beratungskompetenzen wurden keine unterrichtet, obschon Gesprächsführung und Beratung einen grossen Teil unserer Tätigkeit ausmachen.» Auch später, als sie in einem Spital arbeitete, habe sie zur wissenschaftlichen Basis ihres Berufes kaum Zugang gehabt. «Ich war zehn Jahre lang Hebamme
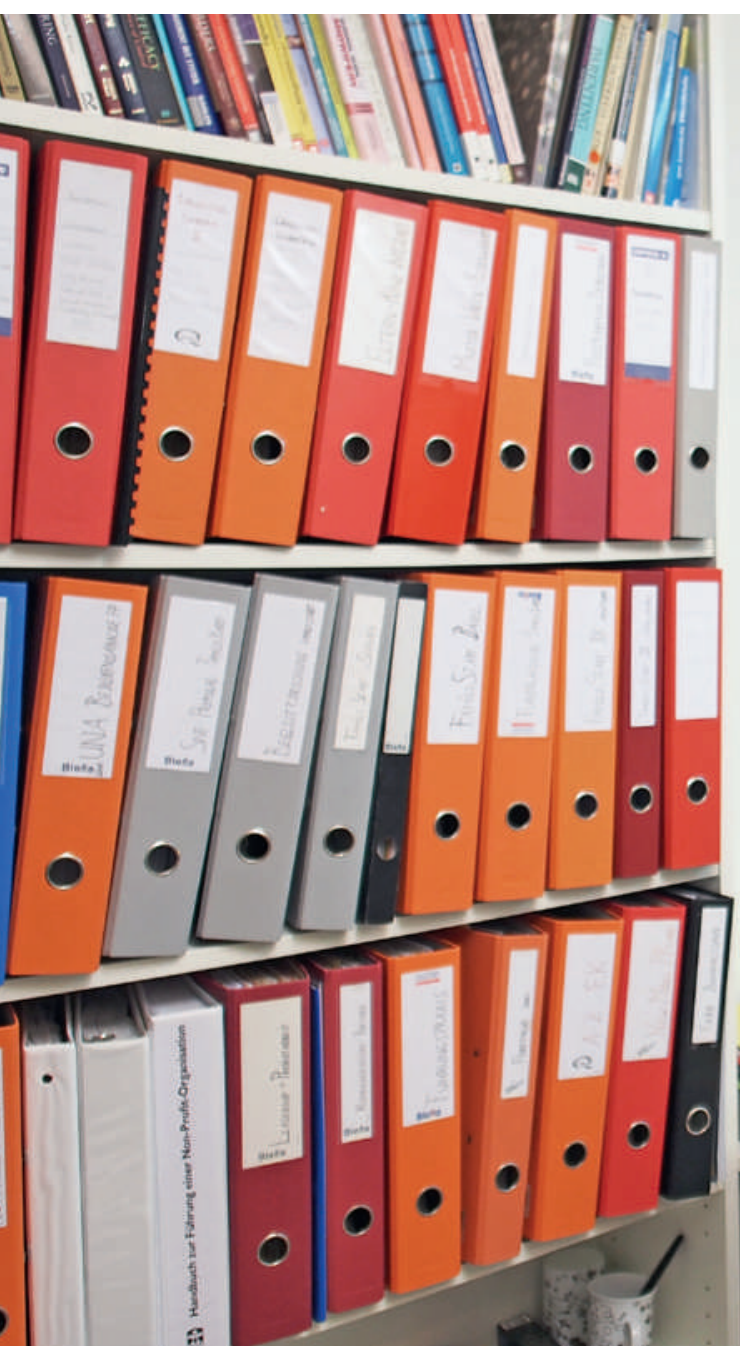

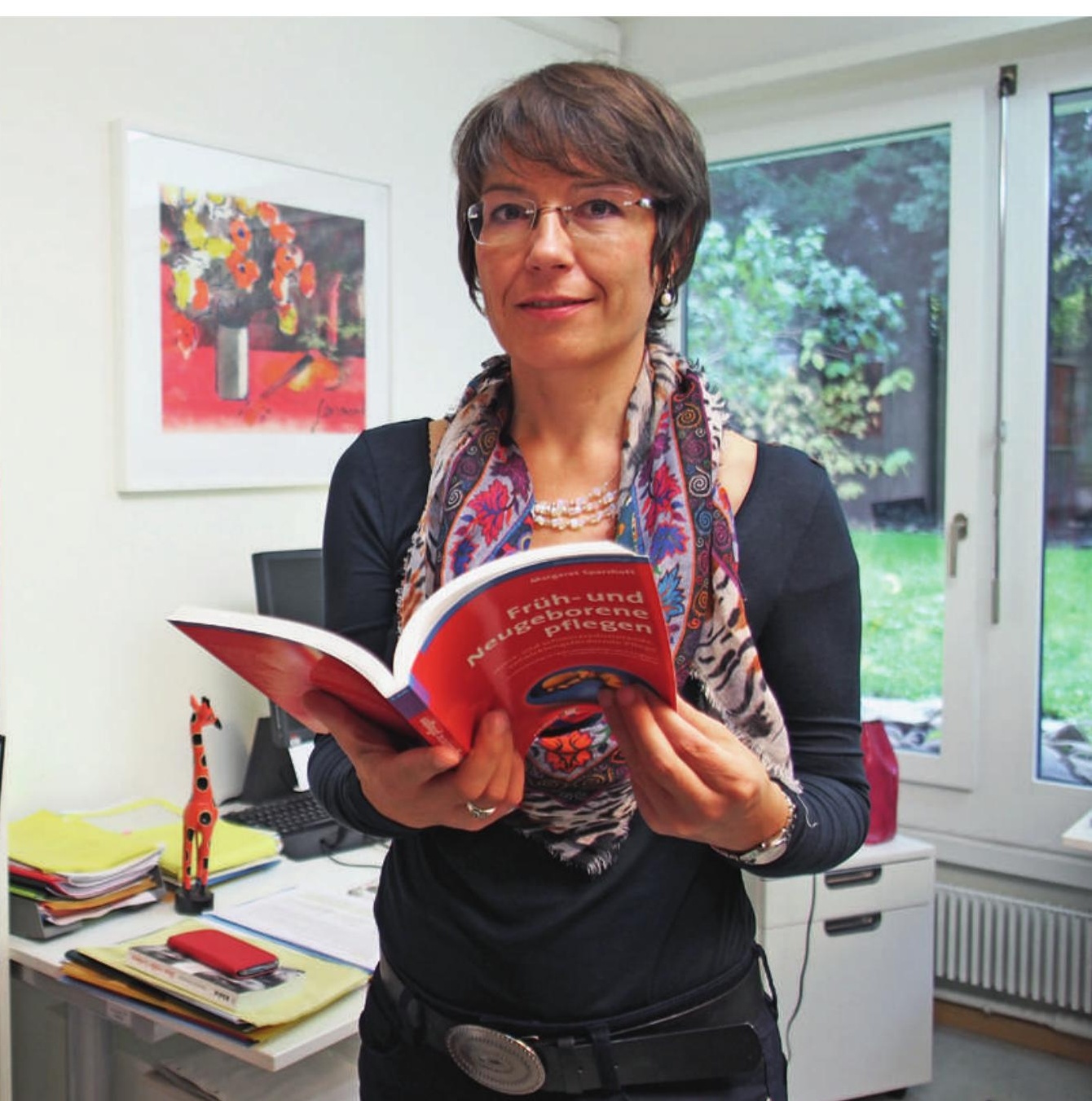


und hatte von wichtigen Studien keine Ahnung.» So auch von einer Studie aus den Fünfzigerjahren nicht, die das Schreiverhalten von Säuglingen analysiert hatte. Kurth machte das Thema zum Zentrum ihrer Doktorarbeit.

\section{Nachbetreuung im Fokus}

2004 entschied sie sich für den akademischen Weg. «Die Praxis gab ich schweren Herzens auf. Aber ich wollte untersuchen, wie sich die Hebammenpraxis optimieren lässt, damit sie Müttern und Kindern einen möglichst grossen Nutzen bringt.» Bald schon richtete sie ihren Fokus auf die Zeit nach der Geburt, wenn Mutter und Kind aus dem Spital entlassen werden. «Vor der Geburt ist nichts zu teuer, aber nach der Geburt ist es, als ob Mutter und Kind nichts mehr nötig hätten. In die pränatale Diagnostik wird fast unbegrenzt investiert, bei der postpartalen Betreuung und Versorgung wird gespart. Und das obschon man heute weiss, wie wichtig die Faktoren der frühen Kindheit für die gesunde Entwicklung eines Kindes sind. Das ist für mich eine besorgniserregende gesellschaftliche Entwicklung.» Entscheidend sei nicht, wie lange Mutter und Kind nach der Geburt im Spital bleiben würden, sagt Elisabeth Kurth, sondern dass sie eine optimale Betreuung erhalten, die auf die individuellen Bedürfnisse zugeschnitten sei. «Eine Hebamme erfasst, wenn etwas nicht stimmt.» Das könne ein medizinisches Problem beim Kind sein, wie eine Infektion, aber auch eine psychische Krise bei den jungen Eltern: «15 Prozent der Mütter und 10 Prozent der Väter erkranken an einer postpartalen Depression.»

Auch gelte es, die Eltern bei der Säuglingspflege anzuleiten, bei der Frau die Laktation und die Rückbildung der Gebärmutter zu überwachen, Brustentzündungen zu erkennen - und die Familie in einer hochdynamischen und besonders sensiblen Lebensphase zu begleiten. Ein zentrales Thema untersuchte sie im Rahmen ihrer Dissertation: Risikofaktoren und Interaktion von Säuglingsschreien und mütterlicher Müdigkeit in den ersten zwölf Wochen nach der Geburt. Seit der erwähnten Studie aus den Fünfzigerjahren wisse man, dass die tägliche Schreidauer bis sechs Wochen nach der Geburt stetig zunehme: «Dann kann auch ein gesundes Baby bis drei Stunden pro Tag schreien, ohne dass man es trösten kann. Das ist seine Art, zu kommunizieren und Bedürfnisse zu manifestieren.» Zwölf Wochen nach der Geburt sinke diese Zahl dann auf unter eine Stunde pro Tag. Aber eben: Es gibt Säuglinge, die sich nicht an die Durchschnittswerte halten.

«Warum Babys exzessiv schreien, ist immer noch ein Rätsel», sagt Elisabeth Kurth. Klar sei, und das habe sie in ihrer Doktorarbeit belegen können, dass alles, was eine Familie unter Stress setzt, das Risiko erhöhe, dass ein Kind viel schreit. «Das können physische Probleme der Mutter sein wie eine Wundinfektion, psychische Symptome oder eine soziale Be-

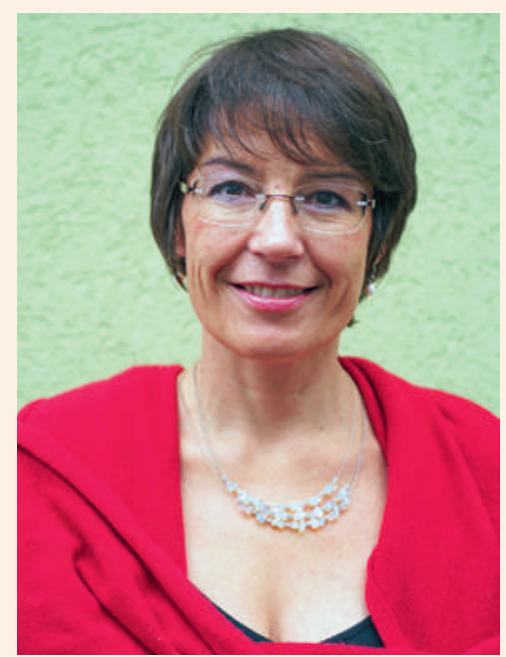

\section{Elisabeth Kurth}

Dr. phil. Elisabeth Kurth wurde 1968 in Spiegel bei Bern geboren, wo sie auch aufwuchs. 1988 machte sie in Köniz die Matura, dann absolvierte sie in Bern die Hebammenschule. 1991 bis 2004 arbeitete sie im Regionalspital Burgdorf. 2001 begann sie ihre Weiterbildung am Institut für Pflegewissenschaft der Universität Basel, die sie 2006 mit dem Master abschloss. Von der Studentin wurde sie zur Assistentin und Doktorandin. 2010 erwarb sie als erste Hebamme an einer Schweizer Universität den Doktortitel. 2010 bis 2014 war sie Dozentin und Forscherin am Institut für Hebammen der ZHAW in Winterthur. Dort ist sie weiterhin als Lehrbeauftragte tätig. Ab 2012 war sie in Basel Präsidentin und Geschäftsführerin des Hebammen-Netzwerks «FamilyStart». Den Job als Präsidentin hat sie inzwischen abgegeben, dafür arbeitet sie seit ein paar Monaten wieder praktisch als Hebamme. Sie ist assoziierte Mitarbeiterin am Schweizerischen Tropen- und Public Health-Institut. Elisabeth Kurth lebt in Kleinbasel.

lastungssituation, die gerade bei Familien mit Migrationshintergrund zu mehr Schreiproblemen führt.»

\section{Dr. Hebamme}

Als Hebamme in der Schweiz einen Doktortitel zu erwerben, war 2010 etwas Exotisches - und ist es immer noch. «Es war schon nur eine grosse Herausforderung, ein Dissertationskomitee zusammenzustellen. Weil es in der Schweiz so etwas bisher nicht gegeben hatte, wurde eine Hebammen-Professorin aus San Francisco zugezogen. Die Hauptbetreuung lag bei einer Basler Medizinerin.» Das Publikumsinteresse an ihrer öffentlichen Prüfung sei dann so gross gewesen, dass man den Raum habe wechseln müs- 
sen. Dass Elisabeth Kurth die erste «Frau Doktor Hebamme» einer Schweizer Universität wurde, war jedoch nicht bloss ein persönlicher Erfolg, sondern ein eigentliches Signal in der Gesundheitspolitik. «Das war ein Meilenstein für den ganzen Berufsstand. Es ist wichtig, dass wir Ausbildung, Qualitätssicherung und Forschung in den eigenen Händen haben», sagt Kurth, «sonst werden wir Hebammen immer mehr in eine Assistenz-Funktion gedrängt, wird die Diskrepanz zwischen ärztlichen und pflegenden, betreuenden Funktionen immer grösser.»

Es gehe um Anerkennung, Eigenverantwortung und interprofessionelle Zusammenarbeit. «Das hat auch ökonomische Vorteile: So zeigt eine kürzlich erschienene Cochrane-Review, dass es bei gesunden Frauen, die eine Hebammen-geleitete statt ärztlich geleitete Geburt haben, weniger medizinische Interventionen gibt, dafür aber eine grössere Zufriedenheit bei den Frauen. Kommt es zu Komplikationen, ist die interdisziplinäre Teamarbeit das A und O.»

Ein Riesenschritt auf dem Weg der Professionalisierung sei 2008 der Wechsel von der Diplomausbildung zum Fachhochschulstudium mit Bachelor und eine Helpline, und falls nötig können wir innerhalb von Minuten den nationalen Telefondolmetschdienst beiziehen. Es freut mich, dass wir so einen Beitrag zur Chancengleichheit leisten können. Und: dass die Familien dank uns mehr Sicherheit und weniger Stress haben.»

In ihrer Doppelfunktion macht Elisabeth Kurth interessante Erfahrungen auch bezüglich der erwähnten Anerkennung: «Wenn ich als Wissenschaftlerin auftrete, werde ich ernst genommen. Bin ich aber als freipraktizierende Hebamme unterwegs, merke ich beispielsweise, wie wenig Know-how Ärzte uns Hebammen manchmal zutrauen. Ein Kulturwandel ist im Gang, aber es bestehen immer noch zu grosse Unsicherheiten, was unsere Kompetenzen sind.»

Der Wiedereinstieg in die Praxis sei ihr nach zehn Jahren gut gelungen, erzählt Kurth, «am meisten Respekt hatte ich vor der Blutentnahme bei den Babys, ich musste mich wieder daran gewöhnen, sie in die Ferse zu stechen.» Ihre theoretischen Kenntnisse seien ihr gerade hier aber zu Hilfe gekommen: «Aus der Forschung weiss ich, dass ein wenig GlukoseLösung oder Muttermilch vor dem Stich bewirkt,

\section{«Warum Babys exzessiv schreien, ist immer noch ein Rätsel.»}

Master gewesen, «wobei es nicht darum ging, einfache Handlungen wie Fiebermessen zu theoretisieren, sondern eben darum, Forschungsevidenz zu erarbeiten und zu vermitteln, die für die Praxis relevant ist.»

\section{FamilyStart}

Heute pendelt Elisabeth Kurth auch selber wieder zwischen Forschung und Praxis hin und her. Etwa 20 Prozent ihres beruflichen Engagements widmet sie seit diesem Frühjahr der Nachbetreuung von Wöchnerinnen und ihren Familien, mehrheitlich mit Migrationshintergrund. Mit ihrem Hebammenvelo ist sie vor allem in Kleinbasel unterwegs, wo sie auch wohnt. «Jede Frau hat eine gute Betreuung zugute, auch eine Eritreerin in einem Flüchtlingsheim.» Koordiniert werden die Einsätze vom Netzwerk «FamilyStart»*, das Kurth gegründet hat und heute noch leitet. «In Basel sind wir etwa 35 Hebammen, die sich untereinander gut koordinieren und engen Kontakt pflegen zu Geburtskliniken, Kinderund Frauenärzten, der Stillberatung und Mütter- und Väter-Beratungsstellen. So haben Eltern auch nach der Geburt immer eine verlässliche Ansprechstelle.

www.gesundheit.zhaw.ch/ familystart dass beim Säugling Endorphine ausgeschüttet werden, die seine Schmerzempfindlichkeit senken.»

\section{Und persönlich?}

Über ihre Arbeit spricht Elisabeth Kurth eindeutig lieber als über sich selbst. Trotzdem: Bei einer Frau, die selber bei über 500 Kindern Geburtshilfe geleistet hat und sich auch wissenschaftlich mit dem Thema jeden Tag auf verschiedenen Ebenen auseinandersetzt, drängt sich die Frage nach eigenen Kindern auf. Gerade in dieser Zeit, in der überall das Lied «Ihr Kinderlein kommet» ertönt. «Gut, ich habe letzthin eine 47-jährige Wöchnerin betreut», antwortet Kurth mit einem Schmunzeln, «für mich selber aber habe ich das Thema allein aus Altersgründen ad acta gelegt.»

Und sonst? Sie koche gerne und freue sich über ein gemütliches Essen mit Freunden, sagt sie. Im Sommer bade sie gern im Rhein, im Winter liebe sie Wellness und Sauna. Auch spiele sie Viola da Gamba. Der Jahreswechsel sei für sie «eine Zeit, um zur Ruhe zu kommen». Eine Bemerkung, die aufhorchen lässt: Gestresst wirkt die vielbeschäftigte Frau Doktor Hebamme nämlich keineswegs.

\section{Die nächste «Begegnung mit ...»}

Am Ende jeden Monats stellt die Schweizerische Ärztezeitung eine Persönlichkeit vor, die sich im Gesundheitswesen engagiert. Im Januar schildert Daniel Lüthi seine Begegnung mit Mechtild Willi Studer, Leiterin Pflegemanagement im Schweizer Paraplegiker-Zentrum Nottwil. 